\title{
An advanced III-V-on-silicon photonic integration platform
}

\author{
Yingtao Hu, Di Liang* and Raymond G. Beausoleil
}

\begin{abstract}
In many application scenarios, silicon (Si) photonics favors the integration of III-V gain material onto Si substrate to realize the on-chip light source. In addition to the current popular integration approaches of III-V-on-Si wafer bonding or direct heteroepitaxial growth, a newly emerged promising solution of epitaxial regrowth on bonded substrate has attracted a lot of interests. High-quality III-V material realization and successful laser demonstrations show its great potential to be a promising integration platform for low-cost, high-integration density and highly scalable active-passive photonic integration on Si. This paper reviews recent research work on this regrowth on bonded template platform including template developments, regrown material characterizations and laser demonstrations. The potential advantages, opportunities and challenges of this approach are discussed.
\end{abstract}

Keywords: Si photonics; III-V-on-Si laser; photonic integration; epitaxy regrowth

Hu YT, Liang D, Beausoleil RG. An advanced III-V-on-silicon photonic integration platform. Opto-Electron Adv 4, 200094 (2021).

\section{Introduction}

With global data traffic growing exponentially, the electrical interconnects have hit a brick wall to satisfy the huge demand of high speed, energy-efficient and cost-effective data transmission in next-generation data centers, high-performance computers, and many emerging applications. By leveraging the mature complementary metal oxide semiconductor (CMOS) manufacturing processes, silicon $(\mathrm{Si})$ photonics has experienced blooming developments over the past 20 years. It now holds the great promise to overcome the data transmission bottleneck problem by providing fast, reliable, and low-cost optical links. However, due to the intrinsic nature of indirect bandgap of Si material, a laser source on $\mathrm{Si}$ is the key challenge for Si photonics. Approaches by engineering group IV materials or their alloys have resulted in $\mathrm{Ge}$ and Ge-alloy ( $\mathrm{GeSi}$ and $\mathrm{GeSn}$ ) lasers on $\mathrm{Si}^{1-3}$, but the laser performances are still far behind the requirements for practical applications. A number of approaches by integrating group III-V materials or devices onto a $\mathrm{Si}$ substrate have been widely developed. The hybrid integration which directly assembling finished III-V laser die onto Si photonic chip with flip-chip ${ }^{4}$ technique is the current mainstream commercial solution. This approach has the advantages of independent III-V and Si optimization and qualification and high light-emitting efficiency. But it requires precise assembly to couple light between the two which leads to high packaging cost and it's not suitable for low-cost mass manufacturing and dense integration. A more favorable approach, the heterogeneous integration, is to transfer III-V optical gain material on patterned silicon-on-insulator (SOI) wafer by wafer bonding and then process the large-scale wafer with CMOS compatible fabrication ${ }^{5}$. This approach allows low-loss evanescent optical coupling from III-V active medium to Si photonic circuits which would reduce

Hewlett Packard Labs, Hewlett Packard Enterprise, 820 N McCarthy Blvd, Milpitas, CA 95035, USA.

*Correspondence: D Liang, E-mail: di.liang@ieee.org

Received: 7 December 2020; Accepted: 5 June 2021; Published: 25 September 2021

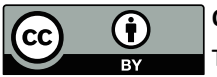

Open Access This article is licensed under a Creative Commons Attribution 4.0 International License

To view a copy of this license, visit http://creativecommons.org/licenses/by/4.0/.

(C) The Author(s) 2021. Published by Institute of Optics and Electronics, Chinese Academy of Sciences. 
packaging costs. It also has the advantage to bond different epitaxial materials onto a single $\mathrm{Si}$ substrate ${ }^{6}$ and is able to realize dense integration. A third category is the direct epitaxial growth of III-V layers on Si or SOI substrate to realize monolithic integration. There are two main approaches among this category. One is the selective area growth of a relative small volume of III-V materials on pre-patterned ${ }^{7,8}$ or oxide-hollow-template-assisted $^{9-11}$ silicon substrate. Excellent epitaxial material have been achieved and room temperature distributed feedback (DFB) lasers ${ }^{7}$, comb lasers ${ }^{8}$ or microcavity lasers have been successfully demonstrated with good performance. However, it still requires extensive R\&D to enable electrically pumped lasers and reliability for the selective growth approach. The other approach is planar growth on full wafers by using intermediate buffer layers to minimize dislocations propagating into the active region. As it's potentially to be the ultimate solution for very high density integration, it has regained huge interest $^{12-17}$. As a result of newly developed novel substrate patterns, intermediate buffer layers, and the use of defect-tolerant active regions, e.g., quantum dot (QD), significant progress has been achieved with the demonstrations of lasers with high efficiency ${ }^{13}$, low threshold ${ }^{14}$, direct modulation ${ }^{16}$ and good lifetime ${ }^{18}$. However, several other critical issues associated with this approach currently include potentially more challenges in heteroepitaxy on a SOI substrate over bulk Si ones; difficulty in achieving efficient light coupling from the III/V active region to the Si waveguide due to inevitable several $\mu \mathrm{m}$ thick buffer layers; and extra optical loss when light propagates in the dense dislocation zones. The fourth approach is transfer printing which is transferring III-V gain materials or devices on Si photonic chips by using a soft elastomeric PDMS stamp. While the proof-ofconcept of demonstrations of wideband-emission LEDs and high-speed photodetectors show great potential to be a cost-effective III-V on Si integration technology, it still requires extensive research to address challenges include transfer yield, degraded bonding quality for large area transfer printing and alignment issue. Also large cost to partially or completely fabricate the III-V components on its 50 or $75 \mathrm{~mm}$ native substrate is still inevitable.

More recently, an advanced heterogeneous integration approach by combining the advantages of direct monolithic epitaxy and wafer bonding approaches has gained increasing research interest. By using wafer bonding to bring InP-based active material onto a Si substrate and then epitaxially growing InP onto the bonded III-V material to bury the pre-defined mesa, researchers from NTT have developed a lateral p-i-n for lateral-currentinjection heterostructure membrane laser ${ }^{19}$. Very impressive demonstrations of high performance lasers show great potential of this scheme for compact and high efficiency lasers on $\mathrm{Si}^{20-23}$. However, it requires a relatively complex and unconventional process to make $\mathrm{p}$ - and $\mathrm{n}$ doping regions and to form a lateral $\mathrm{p}$-i-n diode structure through an extra regrowth step. This unique process is not readily available in most commercial epitaxial growers. Coincidentally, the advanced heterogeneous integration of epitaxial regrowth of III-V onto III-V-on-Si bonded substrate has been reported from several other research groups while they mainly focus on conventional vertical p-i-n-type of diode lasers on silicon. A research group form Sophia University has demonstrated a double heterostructure (DH) laser $^{23-25}$ and a multiquantum well (MQW) laser ${ }^{26,27}$ on $\mathrm{Si}$ substrate with this combined approach. We have studied the quality of regrowth material extensively and demonstrated FabryPerot (FP) lasers on SOI substrate with light easily coupled to $\mathrm{Si}$ waveguides ${ }^{28-30}$. Researchers from III-V Lab have reported their results by using epitaxial growth on $\mathrm{InP}_{-} \mathrm{SiO}_{2} / \mathrm{Si}$ bonding substrate as well ${ }^{31,32}$. These proof-of-concept demonstrations of lasers on Si with this advanced heterogeneous integration approach show its great potential. In this paper, we review the aforementioned research work on this advanced heterogeneous integration platform, particularly formation of conventional vertical $p-i-n$ diode laser structure via thick III-V epitaxial growth. An outlook of this young area is discussed at the end of this paper. This paper is organized as follows. First, the developments of the III-V-on-Si bonding templates for epitaxial regrowth are described. Next, the epitaxy regrowth and materials characterizations is provided. Additionally, the performance characterizations of lasers are detailed. Finally, a discussion and a summary of this advanced heterogeneous integration method are given.

\section{Development of III-V-on-Si bonding templates}

The development of III-V-on-Si bonding templates for regrowth is basically a III-V-to-Si wafer bonding process. As the epitaxy temperature of III-V material is normally as high as $650{ }^{\circ} \mathrm{C}$, only a direct wafer bonding technology may be suitable for such regrowth template preparation while the adhesive bonding, e.g., DVS-BCB 
bonding, is not as the decomposition temperature of most of the suitable polymers, which is much lower than the epitaxy temperature. Demonstrations of bonding templates from different research groups are all based on direct wafer bonding with some slight differences between each other. Figure 1 is a schematic drawing to summarize the major process steps to form such a vertical p-i-n diode laser structure on Si. We highlight the differences in bonding techniques, epitaxy and post-epi fabrications from different research groups.

The Si substrate could be pure Si or SOI wafer while the later provides the possibility to create $\mathrm{Si}$ waveguides or other silicon photonic structures under the III-V tem- plate layer. To realize photonic structures in $\mathrm{Si}$ underneath the III-V template layer, pre-patterning on the SOI wafer is necessary before the wafer bonding. In our work, we also patterned vertical outgassing channels (VOC) in Si to avoid bonding defect formation, i.e., gas voids, due to gas product as a result of polymerization reactions at bonding interface. III-V Lab used a $200 \mathrm{~nm}$ thermal oxide on top of $\mathrm{Si}$ wafer as a hydrogen reservoir to allow diffusion of gas product and subsequently improve bonding quality. While there is no patterns or oxide layer in Sophia University's bonding method but both $\mathrm{Si}$ substrate and template wafer underwent a hydrophilic treatment by $\mathrm{H}_{2} \mathrm{SO}_{4}$ cleaning. The III-V template

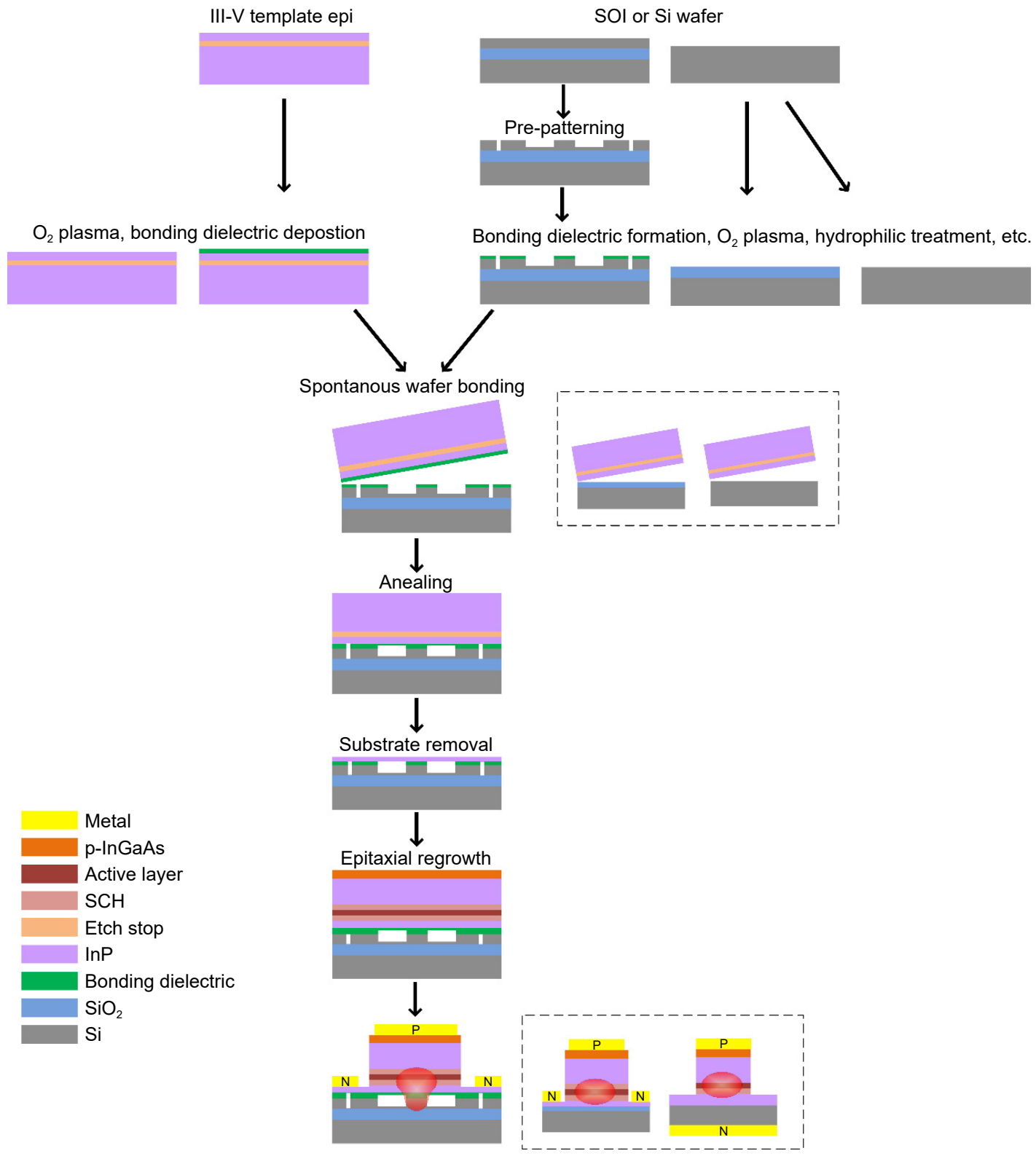

Fig. 1| A schematic drawing of the process flow for the advanced heterogeneous integration by using regrowth on III-V-on-Si bonding template. 
epitaxy wafer basically includes a bonding layer and a etch stop layer on a native substrate. For the InP based templates, the bonding layer is InP and is normally $\mathrm{n}$ doped to act as a contact layer of an active device. The InGaAs etch stop layer is used to selectively etch off InP substrate in the $\mathrm{HCl}$-based solution, and then result in an ideal surface for regrowth once it is selectively removed. After both top surfaces of $\mathrm{Si} / \mathrm{SOI}$ and III-V template wafers are cleaned and activated with $\mathrm{O}_{2}$ plasma, bonding dielectric deposition or $\mathrm{H}_{2} \mathrm{SO}_{4}$ solution treatment, the III-V wafer is bonded to $\mathrm{Si} / \mathrm{SOI}$ wafers. By applying pressure and annealing at $300-400{ }^{\circ} \mathrm{C}$, strong covalent bonds are formed. The next is to remove the III-V substrate and etch stop layer. Upon selectively removing the etch stop layer to expose the n-InP template layer, the bonding template is ready for epitaxial regrowth. The pictures from left to right in Fig. 2 show the fabricated InP-on-Si bonded wafers from our work, III-V Lab and Sophia University's work, respectively. We carried out the bonding with a quarter of 2 inch InP wafer to a SOI wafer, while III-V Lab and Sophia University realized the growth templates by bonding a 4 inch full InP wafer on a 6 inch $\mathrm{SiO}_{2} / \mathrm{Si}$ wafer, and a 2 inch full InP wafer on a 2 inch Si wafer, separately. All bonded growth templates were verified to be robust enough for elevated epitaxial growth temperature.

After the development of bonded growth templates, the following is epitaxial regrowth of lattice-matched III$\mathrm{V}$ device layer stack with metalorganic vapor phase epitaxy (MOVPE). Finally, the wafer experienced subsequent processing of III-V laser devices fabrication. We note that the type of Si-based substrate (SOI or $\mathrm{Si}$ ) and the specific design of III-V layers determine the optical mode profiles. By using pre-patterned SOI and thin III-V template layer, a hybrid mode can be realized which enables evanescent optical coupling from III-V active layers to $\mathrm{Si}$. When a pure Si substrate is used, thick n-layer is necessary to allow the optical mode mainly confined in III-V without leaking into the Si substrate. The bonded growth templates developed from these three research groups are suitable for regrowing laser structures and making vertical injection lasers. Considering significant difference between lateral $\mathrm{p}-\mathrm{i}-\mathrm{n}$ diode laser structure in NTT's solution and vertical ones from the rest of 3 groups, we focus on reviewing progress to demonstrate vertical p-i-n lasers on this platform in this paper.

\section{Epitaxial regrowth and material characterizations}

\section{Epitaxial regrowth}

In our work, we conducted the epitaxial regrowth of a laser structure on the bonding template with a MOVPE system in a commercial vendor. The growth temperature is $600^{\circ} \mathrm{C}$. The epitaxy structure, as shown in Fig. 3(a), is similar to previously demonstrated heterogeneous laser structures ${ }^{33}$ with low-loss evanescent coupling to the Si photonic circuits. The InGaAsP-based MQW is designed for light emission at $1.31 \mu \mathrm{m}$. The total epitaxial thickness is $\sim 2 \mu \mathrm{m}$. In III-V Lab's work, an AlGaInAsbased MQW laser with total thickness of $3 \mu \mathrm{m}$ (Fig. 3(b)) was grown at $610{ }^{\circ} \mathrm{C}$ in a MOVPE system. In Sophia University's work, a InGaAsP-based MQW laser structure was grown in a low pressure MOVPE system at an epitaxy temperature of $650{ }^{\circ} \mathrm{C}$. The epitaxy structure is shown in Fig. 3(c), which shows the total regrowth thickness over $2 \mu \mathrm{m}$. Though the laser structure designs are different, the thicknesses are all above $2 \mu \mathrm{m}$ for conventional vertical injection lasers. The introduction of the bonded template eliminated lattice and polarity mismatches between the Si and III/V material, while the thermal mismatch still exists. The impact of this remaining mismatch can be studied by comparing the epitaxy quality on the bonded substrate and that on a native
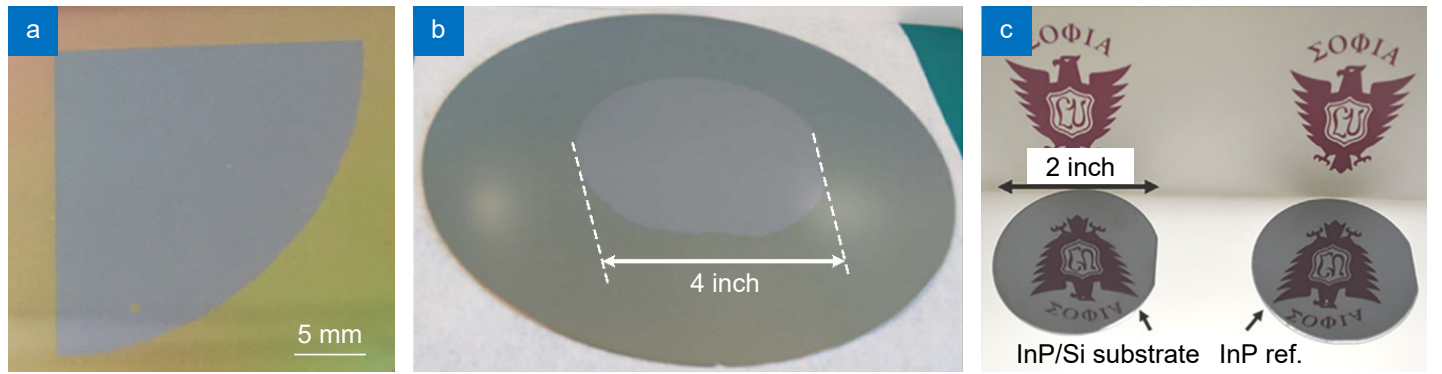

Fig. 2 | Pictures of the fabricated InP-on-Si bonded wafers from HPE, III-V Lab and Sophia University. Figure repoduced with permisson from: (a) ref. ${ }^{29}$, under a Creative Commons Attribution 4.0 International License; (b) ref. ${ }^{31}$, (c) ref. ${ }^{25}$, John Wiley and Sons. 

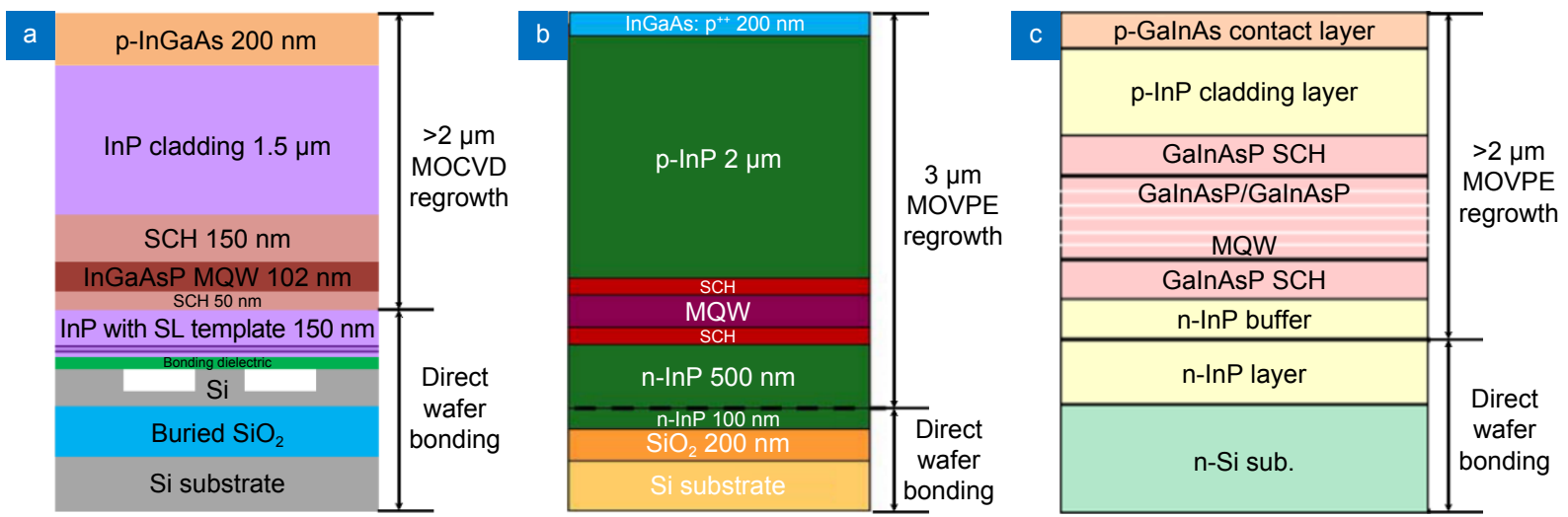

Fig. 3 | Epitaxial regrowth laser structures on bonded templates from HPE, III-V Lab and Sophia University. Figure repoduced with permisson from: (a) ref. ${ }^{29}$, under a Creative Commons Attribution 4.0 International License; (b) ref. ${ }^{32}$, (c) ref. ${ }^{26}$, IEEE.

substrate. All experiments have included the same growth on native InP substrates for comparisons of material quality or device performance.

\section{Surface characterizations}

Surface roughnesses of the regrowth epitaxy were characterized with atomic force microscopy (AFM), we obtained a root mean square (RMS) value of $0.2 \mathrm{~nm}$ while that of III-V Lab's growth is $0.7 \mathrm{~nm}$. Figure 4(a) and 4(b) show the AFM images of the epitaxy regrowth of our work and III-V Lab's work. Particularly, we obtained an identical RMS value of $0.2 \mathrm{~nm}$ on the substance epitaxially grown on the reference InP substrate. This indicated a comparable material quality of subtance epitaxially grown on a bonded substrate versus on a native substrate. Surface quality of the epitaxy material was estimated with Nomarski imaging, as shown in Fig. 4(c), which shows that the surface is smooth while with $2.5 \%$ of bonding caused voids occupancy in Sophia University' $s$ work. More study ${ }^{27}$ shows that the density of bonding caused voids which intuitively represents epitaxy quality affects the lasing threshold current density accordingly.

\section{Dislocation characterizations}

Another routine of characterization is to study material cross-section with transmission electron microscopy (TEM) to investigate possible defects. Figure 5(a) and 5(b) show the cross-sectional TEM images of the MQW epitaxy on the bonded substrate from our work, while Fig. 5(c) is the cross-sectional STEM and Fig. 5(d) is the cross-sectional TEM images from III-V Lab's work. No defect was observed in both TEM specimens (Fig. 5(a) and 5(c)) and MQW layers with good contrast are clearly exhibited in the high-magnification TEM images (Fig. 5(b) and 5(d)) from both work. Particularly in our work, since we were unable to find any threading dislocation (TD) in the epitaxy from cross-sectional TEM imaging on TEM specimens whose plan-view area is around 0.75 $\mu \mathrm{m} \times 15 \mu \mathrm{m}$, we then performed plan-view TEM imaging which provides a relatively large area $(30 \mu \mathrm{m} \times 12$ $\mu \mathrm{m})$ observations. Nevertheless, no TDs but some misfit dislocations were observed with the plan-view TEM. Those misfits were observed at the interface between the upper $\mathrm{SCH}$ and InP cladding layer and within the InP cladding layer close to the interface by tilting
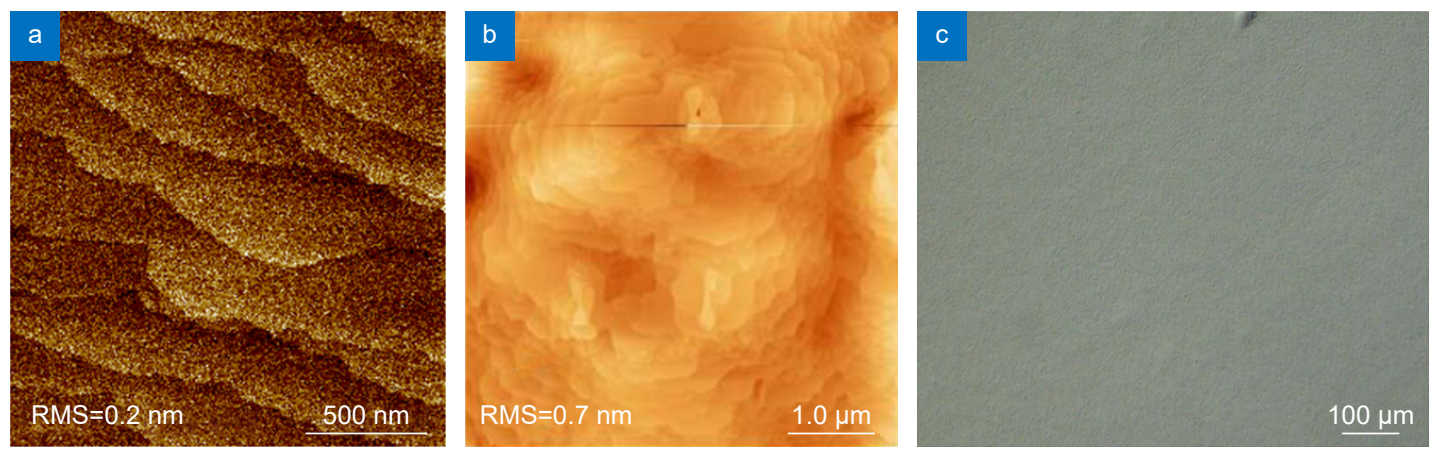

Fig. 4 | AFM images and Nomarski microscope image of the epitaxial regrowth on bonded substrate from HPE, III-V Lab and Sophia University. Figure repoduced with permisson from: (a) ref..$^{29}$, under a Creative Commons Attribution 4.0 International License; (b) ref..$^{32}$, (c) ref. ${ }^{26}$, IEEE. 
cross-sectional TEM specimens. The existence of the misfits is explained as the result of thermal strain in the InP bonding template and the following epitaxy due to the difference in their thermal expansion coefficients and implies that the TDs are far away across the observed plan-view TEM area.
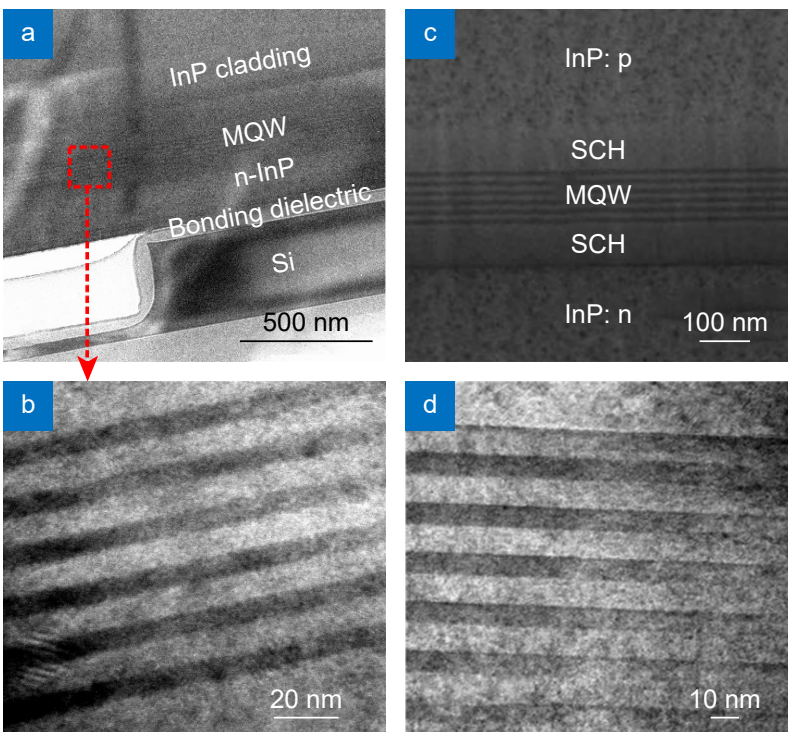

Fig. 5 | Cross-sectional TEM (or STEM) images of the MQW or bulk epitaxy on the bonded substrate from HPE and III-V Lab. Figure repoduced with permisson from: $(a, b)$ ref. ${ }^{29}$, under a Creative Commons Attribution 4.0 International License; (c) ref. ${ }^{32}$, IEEE; (d) ref. ${ }^{31}$, John Wiley and Sons.

Furthermore, we used electron-channeling contrast imaging (ECCI) to quantify the dislocation density in the plan view. Figure 6(a) shows the electron channeling patterns corresponding to the three-beam (400) and (220) imaging conditions that were used. Figure 6(b) shows a representative image with only one TD. A total of 20 TDs were counted in 100 images with a total mapping area of $100 \times 14.5 \times 14.5 \mu \mathrm{m}^{2}$. This led to a dislocation density of $9.5 \times 10^{4} \mathrm{~cm}^{-2}$, only one order of magnitude higher than that of native InP substrates and two orders of mag- nitude lower than state-of-the-art values of conventional monolithic growth with a thick buffer layer ${ }^{34}$. Additional ECCI investigations observed small areas with more concentrated TDs, which were likely caused by bonding voids or dirt particles. We applied the same ECCI process to an InP witness sample for comparison but could not see any TD due to a very low dislocation density for epitaxy on the native substrate.
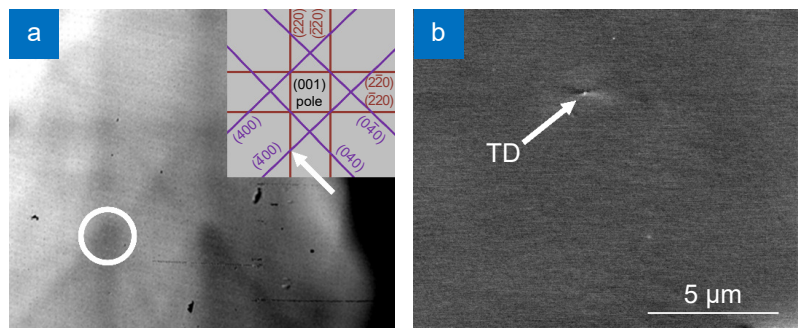

Fig. 6 | (a) The electron channeling patterns corresponding to the three-beam (400) and (220) imaging conditions that were used in $\mathrm{ECCl}$ characterization. (b) A representative $\mathrm{ECCl}$ image with only one TD. Figure repoduced with permisson from ref. ${ }^{29}$, under a Creative Commons Attribution 4.0 International License.

\section{Photoluminescence $(\mathrm{PL})$ measurements}

Figure 7 shows the PL measurements at room temperature (RT) for the epitaxy on both InP and the bonded substrate from the three groups. We found that the measured PL intensity of the MQW from the bonded substrate sample was 2.53 times higher than that from the InP substrate sample, very similar to the 2.7 times higher PL intensity reported in reference ${ }^{21}$. This is mainly because strong reflections from the $\mathrm{Si}$ and buried-oxide layers couple with reflection from the top III/V surface to form a resonance cavity that enhances the PL pump efficiency in the Si substrate sample, as shown in the schematic drawing in the inset of Fig. 7(a). III-V Lab's PL measurements (Fig. 7(b)) show the similar phenomenon that the intensity from epitaxy on bonded $\mathrm{InP} / \mathrm{SiO}_{2} / \mathrm{Si}$ substrate is 5 times higher than that from the
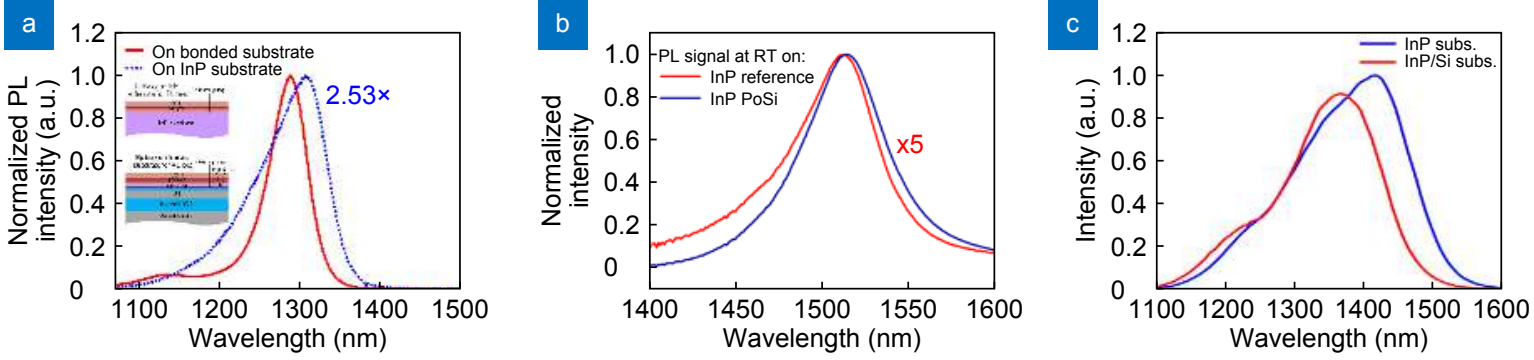

Fig. 7| The PL measurements at room temperature for the epitaxy on both InP and the bonded substrate from HPE, III-V Lab and Sophia University. Figure repoduced with permisson from: (a) ref. ${ }^{29}$, under a Creative Commons Attribution 4.0 International License; (b) ref. ${ }^{32}$, (c) ref. ${ }^{26}$, IEEE. 
InP substrate. As there is no material interface with strong refractive index contrast to cause reflections in Sophia University's case, they reported that the PL intensity from epitaxy on bonded substrate is equivalent to that on the reference InP substrate. The observations of no significant difference on the peak wavelengths, the full widths at half maximum (FWHMs) and PL profiles from all work indicated that the epitaxy quality on the bonded substrate is comparable with that on the InP substrate.

\section{X-ray diffraction (XRD) measurements}

XRD measurement on the epitaxy sample is a method to further investigate the epitaxy quality. Three plots in Fig. 8 are the measured $\omega-2 \theta$ curves both for the epitaxy on bonded and on native substrates for comparison from our work (Fig. 8(a)), III-V Lab (Fig. 8(b)) and Sophia University (Fig. 8(c)). All the three comparisons show that the MQW satellite peaks on both curves exhibit almost identical characteristic signatures, thus indicating good MQW structural similarity and integrity. This means that the epitaxy on bonded substrates has similar high crystal quality to that on native substrates despite total epitaxial thickness is over the critical thickness. Detailed XRD rocking curve analysis and high-resolution omega scans in Fig. 8(a) provide more information of the epitaxy including the strain condition, dislocation density estimation which is a further evidence to our ECCI observations of one order of magnitude higher threading dislocation density (TDD) in the epitaxy on the bonded substrate than that on the native InP substrate ${ }^{29}$.

\section{Laser demonstrations}

\section{III-V-on-SOI FP laser with hybrid waveguide facets} In our work, we treated the regrowth wafer as a conventional heterogeneous wafer and applied the same fabrication procedure to fabricate two types of Fabry-Perot (FP) lasers. One type is FP laser with III-V-on-Si hybrid waveguide facets as front and back mirrors and the other is FP laser with Si waveguide laser facet mirrors. Figure $9(a-c)$ show a microscope image of a FP laser with hybrid facets, schematic of the device cross-section, and SEM of the hybrid facet, respectively. Figure 9(d) and 9(e) show the light-current-voltage (LIV) curves at RT $\left(20^{\circ} \mathrm{C}\right)$ and LI curves up to a stage temperature of $40{ }^{\circ} \mathrm{C}$, both of which are under the pulsed injection mode $(0.5 \mu \mathrm{s}, 0.25 \%$ duty cycle). Large diode voltage in Fig. 9(d) indicates a large series resistance due to fabrication imperfection. The simulated fundamental TE mode profile is shown in the inset of Fig. 9(e). The 1.9-mm-long device starts lasing at $61.8 \mathrm{~mA}$ and emits $4.2 \mathrm{~mW}$ from a single facet under a $120-\mathrm{mA}$ current injection, corresponding to a reasonable threshold current density of $813 \mathrm{~A} / \mathrm{cm}^{2}$ and an overall slope efficiency of $0.14 \mathrm{~W} / \mathrm{A}$. The observation of lasing at approximately $1313 \mathrm{~nm}$ under the pulsed mode at RT (Fig. 9(f)) matches our MQW design well. Figure 9 (g) shows continuous-wave (cw) LI curves up to a stage temperature of $20^{\circ} \mathrm{C}$, with increased thresholds due to device joule heating.

\section{III-V-on-SOI FP laser with Si waveguide facets}

To prove convenient integration with other Si photonic circuits, the FP laser with Si waveguide facets by using two 50- $\mu \mathrm{m}$-long III/V-to-Si tapers to evanescently couple hybrid lasing mode to Si waveguide before reaching polished Si mirrors were demonstrated in our work. Figure 10 (a) shows a microscope image of a FP laser with $\mathrm{Si}$ waveguide facets and a SEM image of a III/V-to-Si taper. Figure $10(b-c)$ show the LIV at RT and the LI curves up to a stage temperature of $35{ }^{\circ} \mathrm{C}$ under the same pulsed mode. The threshold current density of a 2.1-mm-long device with a $2.0-\mathrm{mm}$-long active region was calculated to be $1125 \mathrm{~A} / \mathrm{cm}^{2}$. The simulated fundamental output mode profile at the $\mathrm{Si}$ facet is shown in the inset of
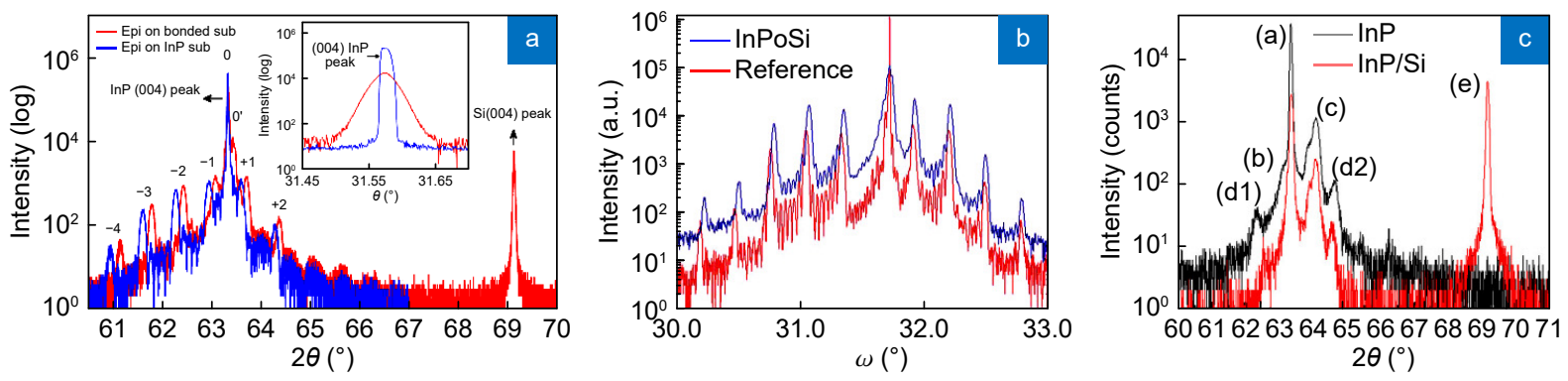

Fig. 8 | XRD measurements on the epitaxy samples that from the three different research groups. Figure repoduced with permisson from: (a) ref. ${ }^{29}$, under a Creative Commons Attribution 4.0 International License; (b) ref. ${ }^{31}$, John Wiley and Sons; (c) ref. ${ }^{27}$, Elsevier. 


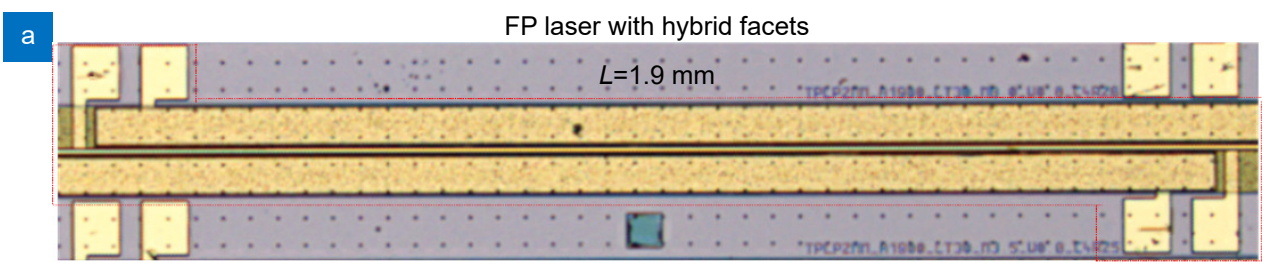

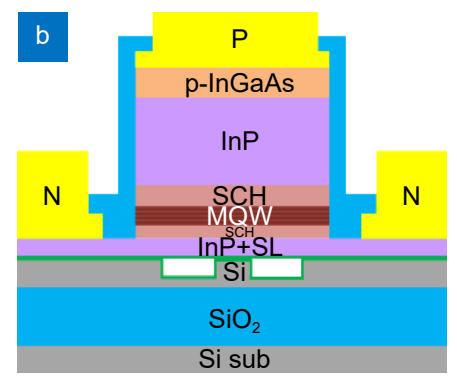

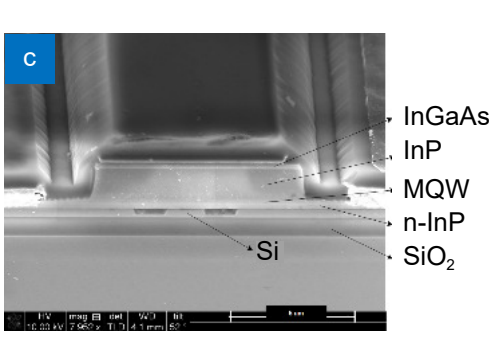

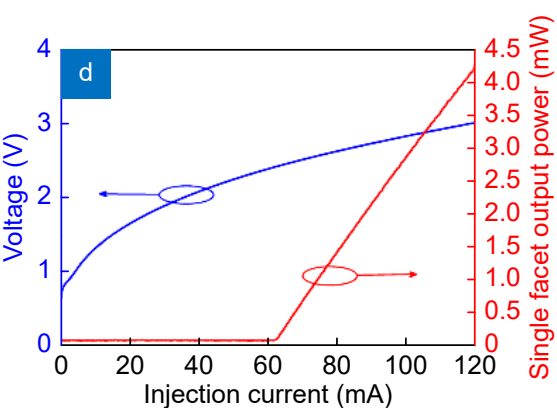

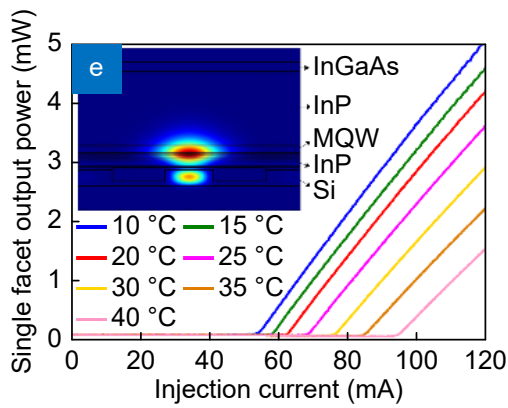

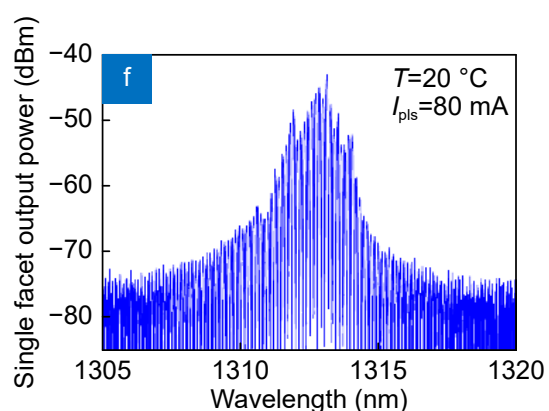

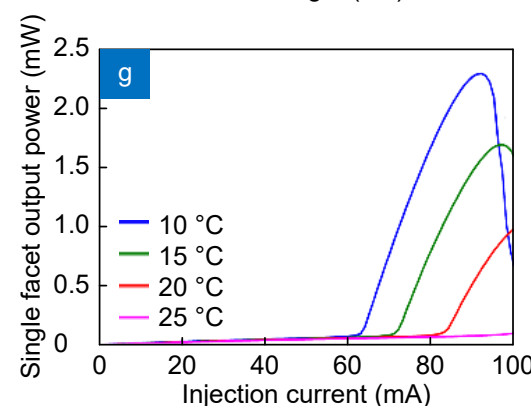

Fig. 9 | (a) A microscope image of a FP laser with hybrid facets. (b) Schematic drawing of the device cross-section and (c) SEM of the hybrid facet. (d) RT pulsed LIV. (e) Pulsed LI up to $40{ }^{\circ} \mathrm{C}$ (inset: mode profile at facets). (f) Device spetrum. (g) $\mathrm{cw} \mathrm{LI} \mathrm{up} \mathrm{to} 25^{\circ} \mathrm{C}$. Figure repoduced with permisson from ref. ${ }^{29}$, under a Creative Commons Attribution 4.0 International License.

Fig. 10(c). We note that multiple fabrication issues including large series resistance and quite high internal loss due to unexpected high p-doping in p-InP cladding, etc., significantly limiting the device performance. However, the demonstration of this laser exhibits the great advantage of readily light coupling from III-V active layer to Si waveguide over other heterogeneous or monolithic works which use pure Si substrate or thick III-V buffer layer where excellent passive optical waveguides in $\mathrm{Si}$ is not employed.

\section{FP laser on Si with III-V facets}

III-V Lab demonstrated broad-area FP lasers on both the bonded growth template (Fig. 3 (center)) and native substrate. Figure 11(a) shows a SEM image of the fabricated laser on bonded substrate. The mesa width is more than $50 \mu \mathrm{m}$ and FP cavity length is $660 \mu \mathrm{m}$. The pulse mode current density-light (J-L) characteristics at $20{ }^{\circ} \mathrm{C}$ of broad-area lasers on bonded and native substrate are shown in Fig. 11(b). The threshold current densities (TCD) at $20{ }^{\circ} \mathrm{C}$ of the laser on bonded substrate and on native substrate are obtained as $400 \mathrm{~A} / \mathrm{cm}^{2}$ and 700
$\mathrm{A} / \mathrm{cm}^{2}$, respectively. The TCD differences could be caused by the difference on the $\mathrm{Zn}$-doping profiles or cleaved facets variations. The measured slope efficiency are 0.092 and $0.095 \mathrm{~W} / \mathrm{A}$ for the laser on bonded substrate and on native substrate, respectively. The facts that the two TCD values are very close and quantum efficiencies are similar indicate that the epitaxy quality grown on a bonded substrate is comparable to that on a native substrate. Figure 11(c) shows the pulse mode J-L characteristics measured at different temperatures up to $50{ }^{\circ} \mathrm{C}$ for the laser on bonded substrate. Figure 11(d) shows the TCD evolution against temperature for the laser on bonded (blue) and on native substrate (red). The laser characteristic temperature $T_{0}$ is extracted to be $53^{\circ} \mathrm{C}$ for both lasers. This result confirms that material quality is preserved for several $\mu \mathrm{m}$-thick epitaxial growth on a bonded substrate.

\section{III-V-on-Si FP laser array}

III-V Lab also demonstrated FP laser array covering 155 $\mathrm{nm}$ spectra band by selective area growth (SAG) on the bonded InP-on-Si substrate ${ }^{35}$. By defining the widths of 


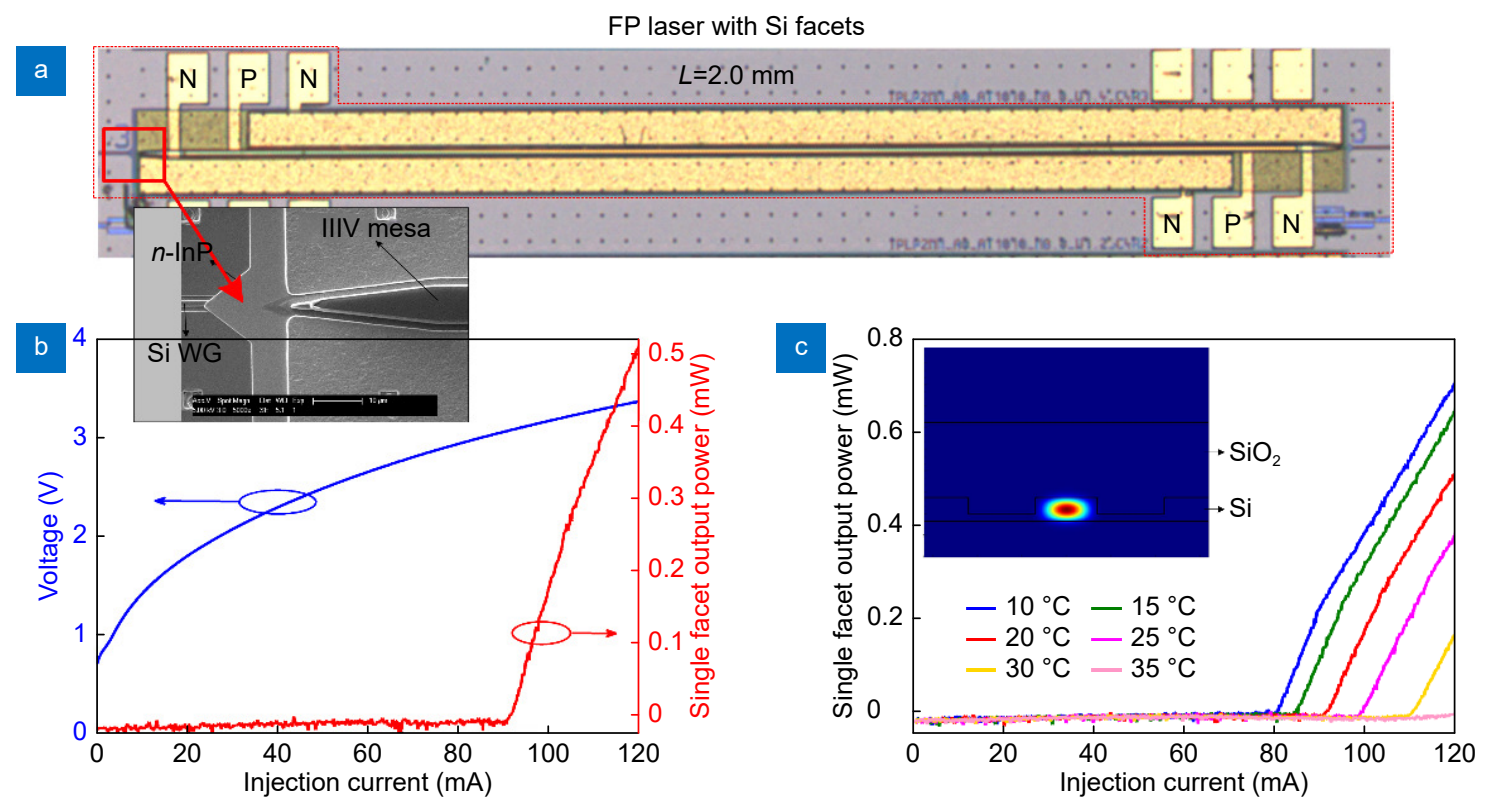

Fig. 10 | (a) A microscope image of a FP laser with Si waveguide facets and a SEM image of a III/V-to-Si taper. (b) RT pulsed LIV (inset: microscope image of the device), (c) pulsed $\mathrm{LI}$ up to $40{ }^{\circ} \mathrm{C}$ (inset: mode profile at facets). Figure repoduced with permisson from ref. ${ }^{29}$, under a Creative Commons Attribution 4.0 International License.
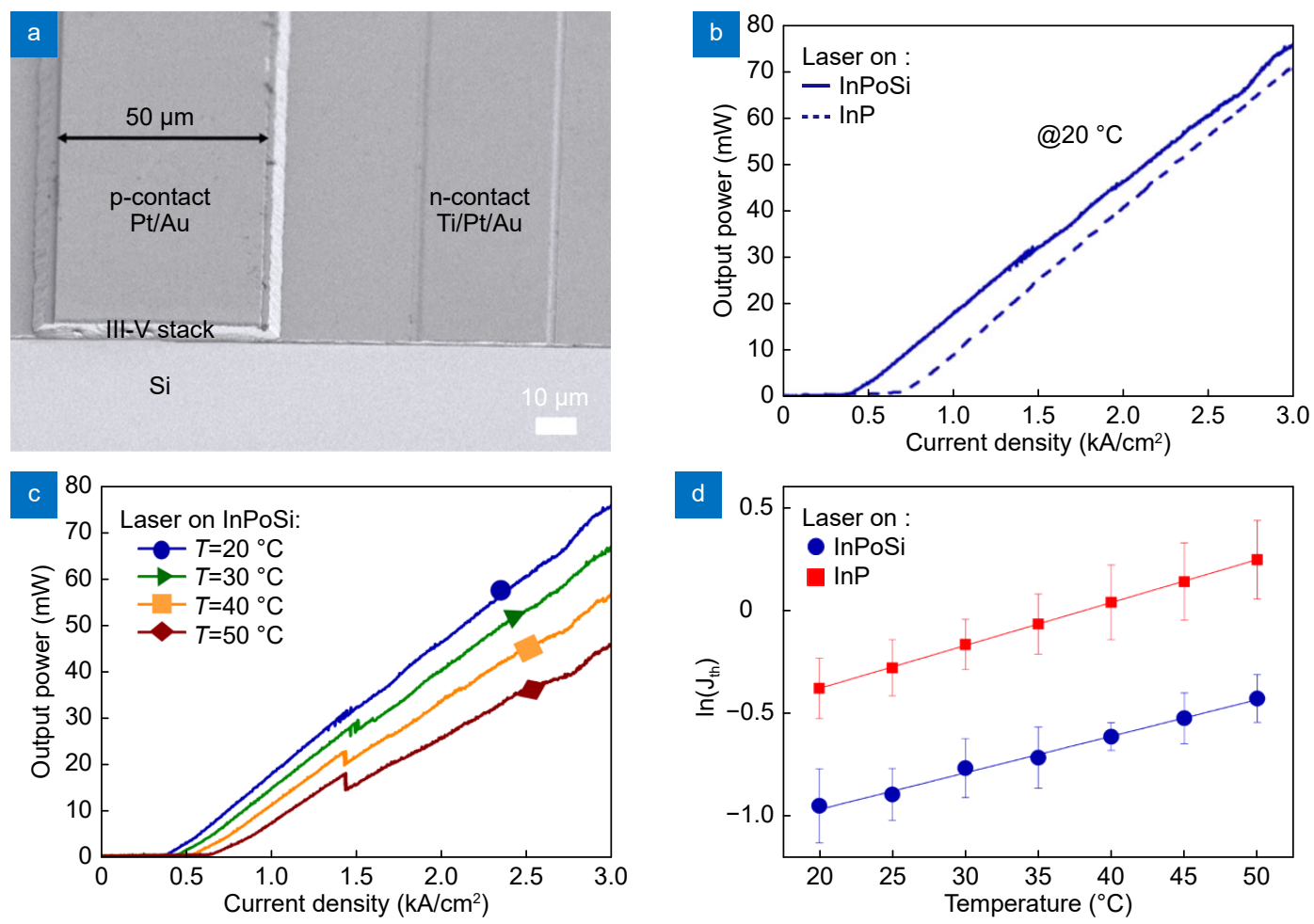

Fig. 11 | (a) SEM image of the fabricated FP laser on InP-on-Si substrate. (b) J-L characteristics in pulse injection mode at $20{ }^{\circ} \mathrm{C}$ : laser on bonded substrate (solid line) and the laser on InP (dash line). (c) J-L characteristics in pulse injection mode measured at different temperatures for the laser on bonded substrate. (d) Threshold current density evolution against temperature for the laser on bonded substrate (blue) and on InP (red). Figure repoduced with permisson from ref. ${ }^{32}$, IEEE.

etching-exposed $\mathrm{SiO}_{2}$ mask that on both sides of fixed width of InP template strips, the thicknesses of the regrown MQW can be tuned accordingly which leads to variations on the MQW photoluminescence emission.
Figure 12(a) shows the measured lasing spectra of five FP lasers based on five different selectively grown MQW active regions. The spectra were measured under $\mathrm{cw}$ operation at a driving current of $100 \mathrm{~mA}$ at $20^{\circ} \mathrm{C}$ for the 

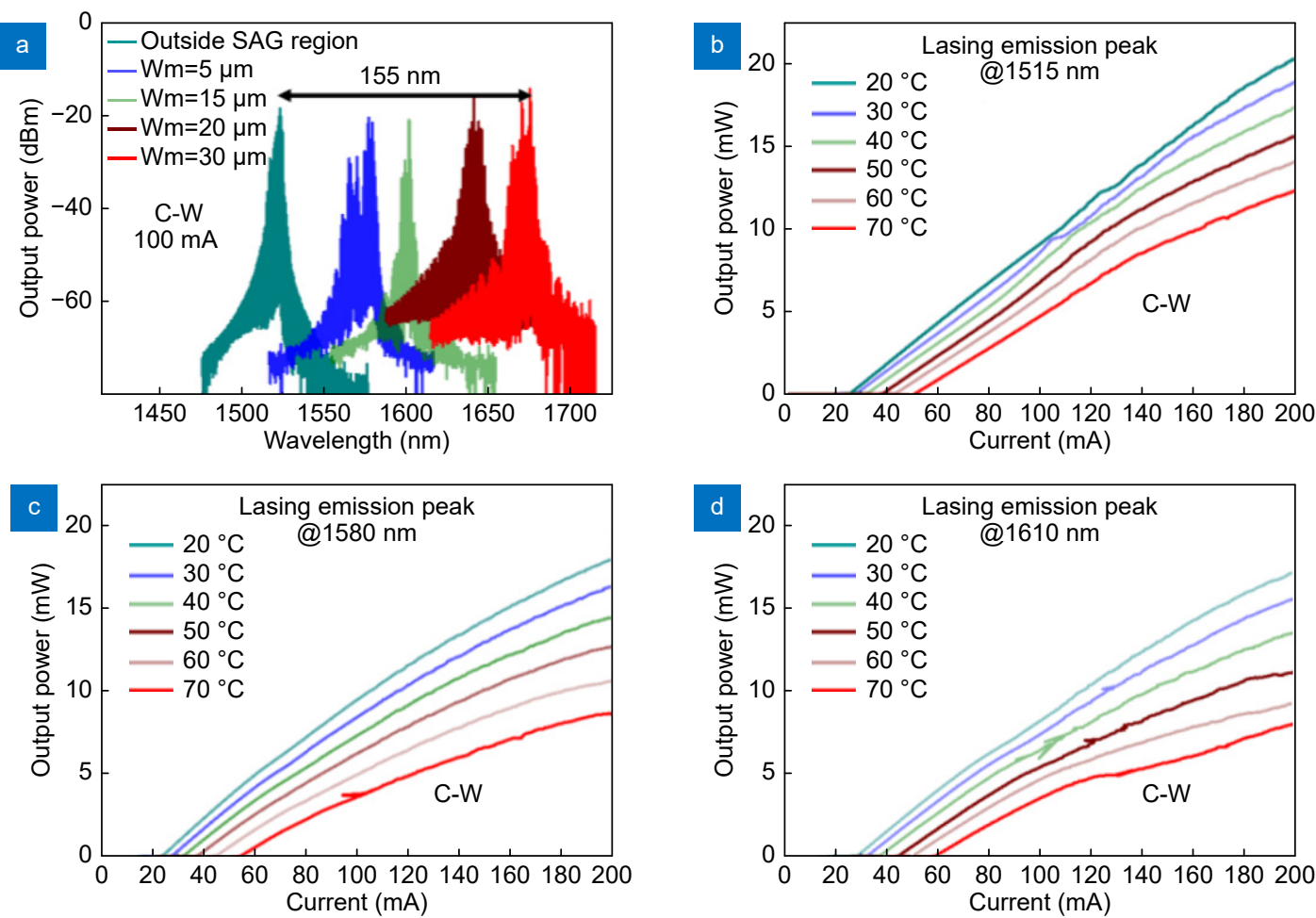

Fig. 12 | (a) Measured lasing spectra of five FP lasers under C-W operation for a driving current of $100 \mathrm{~mA}$ at $20^{\circ} \mathrm{C}$. (b-d) L-I characteristics under C-W operation for different temperatures for the lasers emitting at $1515 \mathrm{~nm}, 1580 \mathrm{~nm}$ and $1635 \mathrm{~nm}$. Figure repoduced with permisson from ref. ${ }^{35}$, IEEE.

$500 \mu \mathrm{m}$-long lasers. It can be seen that a $155 \mathrm{~nm}$ spectra band has been achieved by the single SAG on adjusting the widths of template openings. Figure $12(\mathrm{~b}-\mathrm{d})$ shows the $\mathrm{cw}$ operation LI curves at different temperatures from $20^{\circ} \mathrm{C}$ to $70{ }^{\circ} \mathrm{C}$ of lasers lasing at $1515 \mathrm{~nm}, 1580 \mathrm{~nm}$ and $1635 \mathrm{~nm}$. Decent performance of a maximum of 20 $\mathrm{mW}$ for a $200 \mathrm{~mA}$ driving current and up to $70^{\circ} \mathrm{C}$ for the lasers emitting from $1515 \mathrm{~nm}$ to $1610 \mathrm{~nm}$ were obtained on these SAG lasers. In addition, III-V Lab is adapting their platform to use SOI instead of Si substrate for light coupling into Si waveguides.

\section{FP laser on Si by using Si substrate as one contact} Sophia University has demonstrated InGaAsP-InP bulk DH lasers ${ }^{36,37}$ and MQW lasers ${ }^{26,27}$ on the regrowth on bonded substrates platform. In their laser structure, current was injected through the bonding interface between InP and Si by using Si substrate as the n contact. A typical laser structure is shown in Fig. 13(a). The laser cavities were formed by the manual cleavage of both facets without additional coating on them. The lowest threshold current density for DH lasers at RT and pulse mode is $1800 \mathrm{~A} / \mathrm{cm}^{2}$ lasing at a wavelength of $1.5 \mu \mathrm{m}^{24}$ while the threshold current density is $2850 \mathrm{~A} / \mathrm{cm}^{2}$ for the
MQW lasers. Figure 13(b) shows the typical LI characteristics of the MQW laser at various temperatures. The laser was measured at pulse current mode with pulse width of $0.5 \mu$ s and duty ratio of $0.05 \%$. Figure 13 (c) shows a lasing spectrum for the MQW laser at an injection current density of $J=6.83 \mathrm{kA} / \mathrm{cm}^{2}$, where the lasing wavelength was $1383 \mathrm{~nm}$. They compared lasing characteristics among four different type of lasers: DH bulk laser on bonded substrates and on native substrates, MQW laser on bonded and on native substrates. Figure 13(d) summarized the temperature dependence of the threshold current density for the four types of lasers. We can see that the threshold current density is the same for $\mathrm{DH}$ lasers on the two different substrates while it's quite different for the MQW lasers on the two substrates. The increasing trend in the threshold current density with the temperature is almost the same for the $\mathrm{DH}$ lasers on bonded and native substrates while it's different for the MQW lasers on the bonded and native substrates. As the density of the void in directly bonded InP/Si substrate caused by the growth of the SCH-MQW LD was observed slightly higher than that in the InP/Si substrate with the bulk LD, the difference on the laser performance could be explained as the bonding quality influencing 

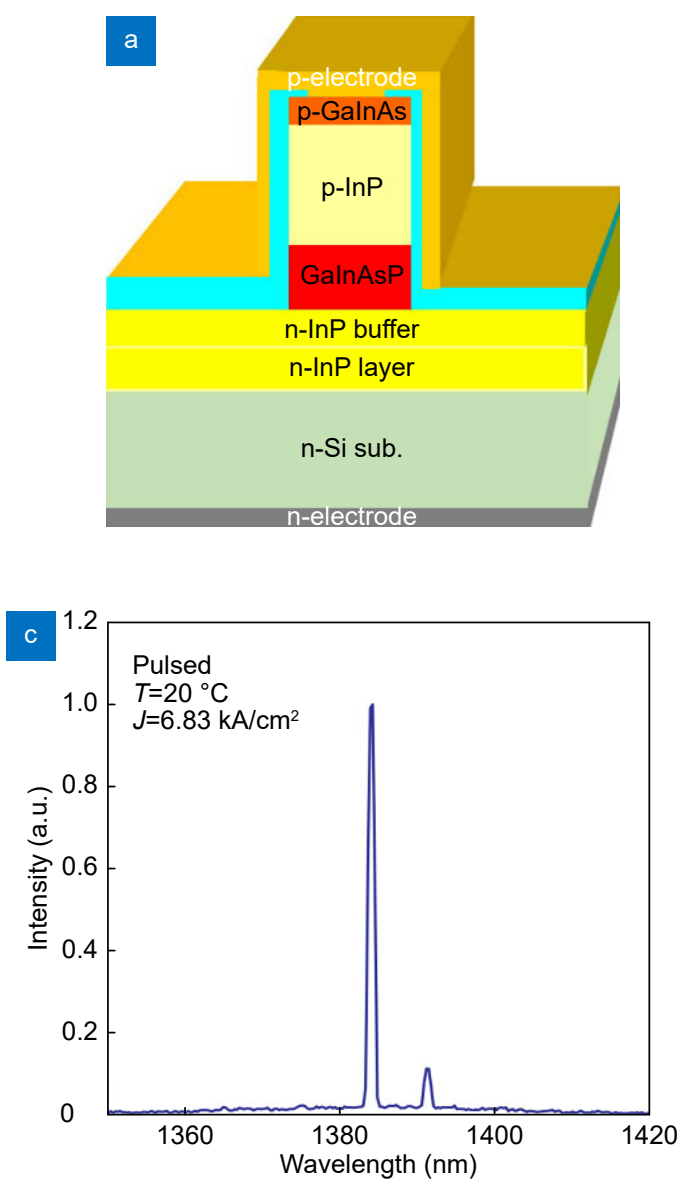
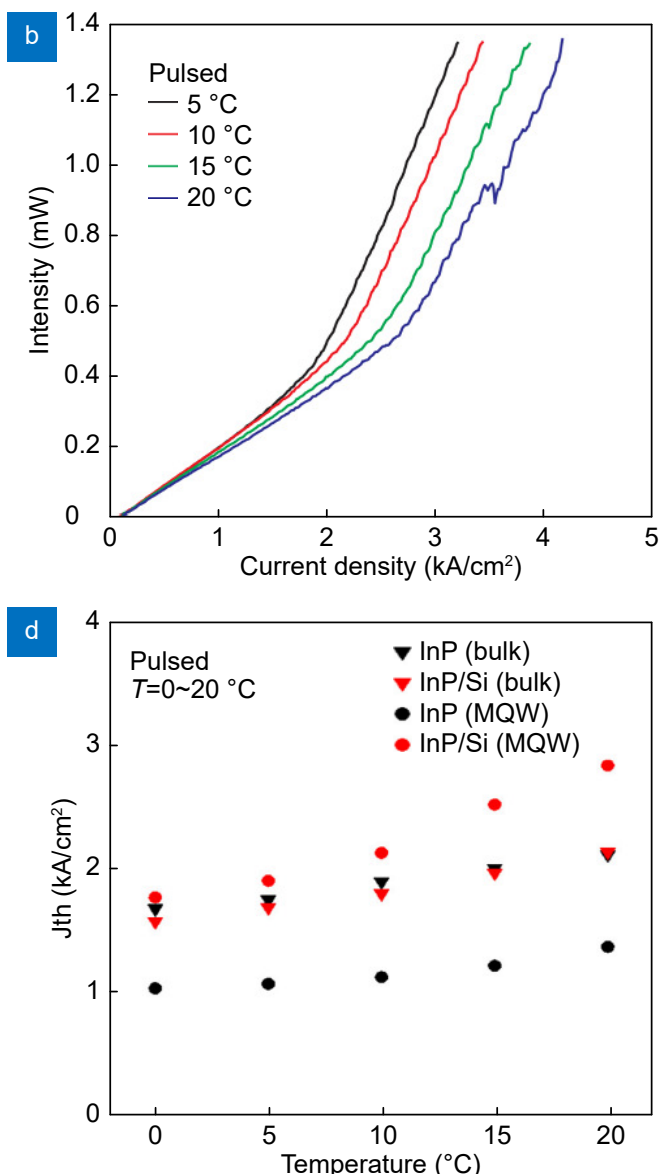

Fig. 13 | (a) A typical laser structure. (b) The typical I-L characteristics of the MQW laser on the bonded substrate at various temperatures. (c) A lasing spectrum for the MQW laser at an input current of $J=6.83 \mathrm{kA} / \mathrm{cm}^{2}$. (d) The temperature dependence of the threshold current density for the $\mathrm{DH}$ bulk lasers and MQW lasers on bonded substrates and on native substraes. Figure repoduced with permisson from: (a) ref. ${ }^{26}$, IEEE; (b, c, d) ref. ${ }^{27}$, Elsevier.

the laser performance significantly as the current has to inject through the bonding interface. Another possible reason might be that $\mathrm{MQW}$-based active region is much more dependent on material quality than that of bulk material. While the similarity of the $\mathrm{DH}$ lasers on the two substrates indicates that epitaxy quality on a bonded substrate that with good bonding quality could be comparable to that on a native substrate.

As a comparison, we summarized the main features including bonding methods, epitaxy characterizations and laser performance of the demonstrations from the reviewed three research groups in Table. 1. It can be seen that III-V lab has achieved the best laser performance while our work demonstrated the ability of light coupling from active region to $\mathrm{Si}$. We believe that more achievements would be inspired by these proof-ofconcept laser demonstrations in the future.

\section{Discussion and summary}

Research progress from different research groups re- viewed above has indicated growing interest and huge potential in this novel concept of regrowth on III-V-onSi bonded substrate. Bonded growth templates with variations in Si side have been successfully developed, and high quality of III-V MQW epitaxy has been confirmed by different characterization methods. Proof-of-concept laser demonstrations further concluded that the electrical and optical properties are well preserved to be comparable to the growth on a native substrate. According to our detailed discussions in our previous work ${ }^{29}$, the regrowth on bonded template method is of huge interest and high value for further R\&D for the following main reasons.

1) High-quality epitaxy with low dislocation density on Si. The approach of epitaxy on bonded template eliminates two of the three major root causes for dislocations: lattice and polarity mismatches between the substrate (e.g., $\mathrm{Si}$ ) and the function material (e.g., III/V) from epitaxial growth. The thermal mismatch between the 
substrate and template material would still cause defects in the regrown materials, but it is measured to be at a significantly low level. According to the aging tests of InAs QD lasers on Si near room temperature ${ }^{18}$, a reduction in the TDD from $10^{8} \mathrm{~cm}^{-2}$ to $10^{6} \mathrm{~cm}^{-2}$ can extend the laser lifetime from a few months to over 100 years. It is reasonable to expect that lasers from the regrowth on the bonded template with even lower dislocation density would eliminate the defect-induced lifetime concerns for all practical applications.

2) It's a generic method for many other heterogeneous material combinations. As the epitaxy quality is high and thickness is up to several $\mu \mathrm{m}$, this growth on bonding template approach could be a generic method for many other heterogeneous material combinations. The substrate could be semiconductors (e.g., Si), dielectrics (e.g., $\mathrm{Si}_{3} \mathrm{~N}_{4}$ ), metals, etc., and the top grown material could be bulk materials, QWs, QDs or other nanostructures. Sequential multiple growths on the same template can be a routine procedure to enable advanced, large wafer-scale, dense photonic integration. A good example in silicon photonics is the integration of light sources, amplifiers, modulators and detectors on a single chip with close proximity and low coupling loss by implementing multiple selective regrowth on a single bonding template instead of bonding three or four types of epitaxial structures on each chip ${ }^{38}$. We believe that various functional materials grown on a single bonded substrate could be emerging as large-scale process.

3) It's cost competitive over the other existing III-Von-Si integration approaches. Table 2 is a qualitative comparison of the production and operation costs of the same diode laser, built using different III/V-on-silicon integration approaches. All costs, including the substrate material (Si and InP substrate), III/V epitaxy, device fabrication (bonding and device fab), chip packaging and operation, are compared separately among the four integration approaches. The marks $(\mathrm{x}, \mathrm{xx}, \mathrm{xxx})$ indicate the relative cost level among the four integration approaches within each column but do not indicate the cost differences over columns. The epitaxy plus bonding approach is enabling material growth on much larger and cheaper substrate than its native substrate. The natural feasibility of multiple regrowth on the same growth template can lead to very high-density integration, thus minimizing the chip size and packaging effort. Therefore, the

Table 1 | A comparison of the main features in the demonstrations from the three research groups.

\begin{tabular}{|c|c|c|c|c|c|c|c|c|c|c|}
\hline \multirow[t]{2}{*}{$\begin{array}{c}\text { Research } \\
\text { group }\end{array}$} & \multicolumn{3}{|c|}{ Bonding methods } & \multicolumn{3}{|c|}{ Epitaxy characterizations } & \multicolumn{4}{|c|}{ Laser performance } \\
\hline & Configuration & $\begin{array}{c}\text { Surface } \\
\text { treatments }\end{array}$ & $\begin{array}{l}\text { Bonding } \\
\text { size }\end{array}$ & $\begin{array}{l}\text { Epitaxy } \\
\text { thickness }\end{array}$ & $\begin{array}{c}\text { Surface } \\
\text { roughness }\end{array}$ & $\begin{array}{l}\text { TDD } \\
\left(\mathrm{cm}^{2}\right)\end{array}$ & $\begin{array}{c}\text { threshold } \\
\text { current } \\
\text { densities } \\
\left(\mathrm{A} / \mathrm{cm}^{2}\right)\end{array}$ & $\begin{array}{c}\text { Slope } \\
\text { efficiency } \\
\text { (W/A) }\end{array}$ & $\begin{array}{l}\text { Temperature } \\
\text { performance }\end{array}$ & $\begin{array}{c}\text { Other } \\
\text { features }\end{array}$ \\
\hline HPE & $\begin{array}{c}\mathrm{InP} / \mathrm{SiO}_{2} / \mathrm{SOI} \\
\text { (patterned) }\end{array}$ & $\begin{array}{l}\mathrm{SiO}_{2} \text { dep., } \\
\text { O2 plasma }\end{array}$ & $1 / 4$ of 2 inch & $>2 \mu \mathrm{m}$ & $0.2 \mathrm{~nm}$ & $9.5 \times 10^{4}$ & $\begin{array}{c}813 \\
1125 \\
\text { (coupled to Si) }\end{array}$ & 0.14 & $\begin{array}{l}\text { Pulsed up to } 40^{\circ} \mathrm{C} \\
\mathrm{CW} \text { up to } 20^{\circ} \mathrm{C}\end{array}$ & $\begin{array}{l}\text { Light coupled into } \\
\text { Si waveguide }\end{array}$ \\
\hline III-V lab & $\ln P / S_{i O} / S i$ & $\begin{array}{l}\text { Thermal } \\
\text { Oxidized } \\
\mathrm{SiO}_{2}\end{array}$ & 4 inch & $3 \mu \mathrm{m}$ & $0.7 \mathrm{~nm}$ & - & 400 & 0.092 & $\mathrm{CW}$ up to $70^{\circ} \mathrm{C}$ & $\begin{array}{l}\text { Laser array } \\
\text { (with } 5 \text { lasers) }\end{array}$ \\
\hline $\begin{array}{c}\text { Sophia } \\
\text { University }\end{array}$ & $\ln \mathrm{P} / \mathrm{Si}$ & $\begin{array}{l}\mathrm{H}_{2} \mathrm{SO}_{4} \\
\text { solution }\end{array}$ & 2 inch & $>2 \mu \mathrm{m}$ & - & - & $\begin{array}{c}1800(\mathrm{DH}) \\
6830(\mathrm{MQW})\end{array}$ & - & Pulsed up $20^{\circ} \mathrm{C}$ & $\begin{array}{c}\text { Metal contact on } \\
\text { Si substrate }\end{array}$ \\
\hline
\end{tabular}

Table 2 | Qualitative comparison of production and operation costs of the same diode laser built on different III/V-on-silicon integration approaches.

\begin{tabular}{|c|c|c|c|c|c|c|c|}
\hline \multirow{2}{*}{$\begin{array}{c}\text { Unit laser area cost } \\
\text { Approach }\end{array}$} & \multicolumn{2}{|r|}{ Substrate material } & \multirow{2}{*}{$\begin{array}{c}\text { III/V } \\
\text { epitaxy }\end{array}$} & \multicolumn{2}{|c|}{ Fabrication } & \multirow[b]{2}{*}{ Packaging } & \multirow{2}{*}{$\begin{array}{l}\text { Operation } \\
\text { (energy \$) }\end{array}$} \\
\hline & $\begin{array}{l}\text { Si/SOI } \\
\text { (12 in) }\end{array}$ & $\ln P$ & & Bonding & $\begin{array}{c}\text { Device } \\
\text { fab }\end{array}$ & & \\
\hline Finished III/V chip packaged with $\mathrm{Si}^{39}$ & None & $x x x(2-4$ in $)$ & $x x x(2-4$ in $)$ & None & $x x x(3$ in) & $x x x$ & $x x$ \\
\hline III/V wafer bonding on $\mathrm{Si}^{40}$ & $x$ & $x x x(2-4$ in $)$ & $x x x(2-4$ in) & $x x$ & $x(12 \mathrm{in})$ & $\mathrm{x}$ & $x$ \\
\hline III/V epitaxy on $\mathrm{Si}^{12-14}$ & $x$ & None & $x x(12$ in) & None & $x(12 \mathrm{in})$ & $x x x$ & $x x$ \\
\hline Wafer bonding plus epitaxy & $\mathrm{x}$ & $x x x(2-4$ in, template epitaxy included) & $x(12 \mathrm{in})$ & $\mathrm{x}$ & $x(12 \mathrm{in})$ & $\mathrm{x}$ & $\mathrm{x}$ \\
\hline
\end{tabular}

x: low, xx: medium, xxx: high 
bonding plus epitaxy approach can be very cost competitive overall.

As direct wafer bonding is critical to this platform based on regrowth on bonded substrate, efficient, reliable and high-quality large area wafer bonding technology is needed for this approach to be adapted in standard CMOS foundries towards commercialization for practical applications. It still needs extensive research and development to conduct multiple and selective regrowth on the template to fully explore the capability and potential of this platform. In addition, investigating options to reuse the III-V substrate for template preparation $^{41}$ is another interesting subject to study for maximizing cost saving nature of this platform. In summary, epitaxy regrowth on bonded substrate is emerging as a novel integration method for III-V-on-Si photonic integration. It is a general approach for combining different materials onto various substrates and particularly suitable for on-chip light source and other functional devices for Si photonics. Current high-quality epitaxy material regrowth and proof-of-concept pulsed and cw lasers demonstrations will inspire more R\&D efforts on this integration platform. It is worth looking forward to in a number of all-in-one photonic integration applications for its low cost, high scalability, and high integration density features.

\section{References}

1. Wirths S, Geiger R, von den Driesch N, Mussler G, Stoica T et al. Lasing in direct-bandgap GeSn alloy grown on Si. Nat Photon 9, 88-92 (2015).

2. von den Driesch N, Stange D, Rainko D, Povstugar I, Zaumseil $\mathrm{P}$ et al. Advanced GeSn/SiGeSn group IV heterostructure lasers. Adv Sci 5, 1700955 (2018).

3. Margetis J, Zhou YY, Dou W, Grant PC, Alharthi B et al. All group-IV SiGeSn/GeSn/SiGeSn QW laser on Si operating up to 90 K. Appl Phys Lett 113, 221104 (2018).

4. Lin SY, Zheng XZ, Yao J, Djordjevic SS, Cunningham JE et al. Efficient, tunable flip-chip-integrated III-V/Si hybrid externalcavity laser array. Opt Express 24, 21454-21462 (2016).

5. Liang D, Roelkens G, Baets R, Bowers JE. Hybrid integrated platforms for silicon photonics. Materials 3, 1782-1802 (2010).

6. Zhang C, Zhang SJ, Peters JD, Bowers JE. $8 \times 8 \times 40 \mathrm{Gbps}$ fully integrated silicon photonic network on chip. Optica $\mathbf{3}$, 785-786 (2016).

7. Wang ZC, Tian B, Pantouvaki M, Guo WM, Absil P et al. Room-temperature InP distributed feedback laser array directly grown on silicon. Nat Photon 9, 837-842 (2015).

8. Wang ZC, van Gasse K, Moskalenko V, Latkowski S, Bente E et al. A III-V-on-Si ultra-dense comb laser. Light Sci App/ 6, e16260 (2017).

9. Wirths S, Mayer B, Schmid H, Lörtscher E, Sousa M et al. Room temperature lasing from monolithically integrated GaAs microdisks on Si. In 2017 Conference on Lasers and ElectroOptics Europe \& European Quantum Electronics Conference (IEEE, 2017); http://doi.org/10.1109/CLEOE-EQEC.2017.808 6366.

10. Wirths S, Mayer BF, Schmid H, Sousa M, Gooth J et al. Roomtemperature lasing from monolithically integrated GaAs microdisks on silicon. ACS Nano 12, 2169-2175 (2018).

11. Mauthe S, Triviño NV, Baumgartner $Y$, Sousa M, Caimi D et al. InP-on-Si optically pumped microdisk lasers via monolithic growth and wafer bonding. IEEE J Sel Top Quantum Electron 25, 8300507 (2019).

12. Chen SM, Li W, Wu J, Jiang Q, Tang MC et al. Electrically pumped continuous-wave III-V quantum dot lasers on silicon. Nat Photon 10, 307-311 (2016).

13. Liu AY, Zhang C, Norman J, Snyder A, Lubyshev D et al. High performance continuous wave $1.3 \mu \mathrm{m}$ quantum dot lasers on silicon. Appl Phys Lett 104, 041104 (2014).

14. Wan YT, Norman J, Li Q, Kennedy MJ, Liang D et al. $13 \mu \mathrm{m}$ submilliamp threshold quantum dot micro-lasers on Si. Optica 4, 940-944 (2017).

15. Liu ST, Wu XR, Jung D, Norman JC, Kennedy MJ et al. Highchannel-count $20 \mathrm{GHz}$ passively mode-locked quantum dot laser directly grown on Si with $4.1 \mathrm{Tbit} / \mathrm{s}$ transmission capacity. Optica 6, 128-134 (2019).

16. Wan YT, Zhang S, Norman JC, Kennedy MJ, He W et al. Directly modulated single-mode tunable quantum dot lasers at 1.3 $\mu \mathrm{m}$. Laser Photon Rev 14, 1900348 (2020).

17. Wan YT, Norman JC, Tong YY, Kennedy MJ, He W et al. 1.3 $\mu \mathrm{m}$ quantum dot-distributed feedback lasers directly grown on (001) Si. Laser Photon Rev 14, 2000037 (2020).

18. Jung D, Herrick R, Norman J, Turnlund K, Jan C et al. Impact of threading dislocation density on the lifetime of InAs quantum dot lasers on Si. Appl Phys Lett 112, 153507 (2018).

19. Matsuo S, Takeda K, Sato T, Notomi M, Shinya A et al. Roomtemperature continuous-wave operation of lateral current injection wavelength-scale embedded active-region photonic-crystal laser. Opt Express 20, 3773-3780 (2012).

20. Matsuo S, Fujii T, Hasebe K, Takeda K, Sato T et al. Directly modulated buried heterostructure DFB laser on $\mathrm{SiO}_{2} / \mathrm{Si}$ substrate fabricated by regrowth of InP using bonded active layer. Opt Express 22, 12139-12147 (2014).

21. Fujii T, Sato T, Takeda K, Hasebe K, Kakitsuka T et al. Epitaxial growth of InP to bury directly bonded thin active layer on $\mathrm{SiO}_{2} / \mathrm{Si}$ substrate for fabricating distributed feedback lasers on silicon. IET Optoelectron 9, 151-157 (2015).

22. Nishi H, Fujii T, Diamantopoulos NP, Takeda K, Kanno E et al. Monolithic integration of an 8-channel directly modulated membrane-laser array and a SiN AWG Filter on Si. In 2018 Optical Fiber Communication Conference (OSA, 2018); http://doi.org/ 10.1364/ofc.2018.th3b.2

23. Aihara T, Hiraki T, Fujii T, Takeda K, Tsuchizawa T et al. 56Gbit/s operations of mach-zehnder modulators using $300-\mu \mathrm{m}$ long membrane InGaAsP phase shifters and $\mathrm{SiN}$ waveguides on Si. In 2019 Optical Fiber Communications Conference and Exhibition (IEEE, 2019); http://doi.org/10.1364/ofc.2019.m4a.3

24. Sugiyama $H$, Nishiyama $T$, Kamada $N$, Onuki $Y$, Han $X$ et al. Low threshold current of GalnAsP laser grown on directly bonded InP/Si substrate. In 2017 Conference on Lasers and Electro-Optics Pacific Rim (IEEE, 2017); http://doi.org/10.1109/ CLEOPR.2017.8119024. 
25. Periyanayagam GK, Nishiyama T, Kamada N, Onuki Y, Shimomura K. Lasing characteristics of $1.2 \mu \mathrm{m}$ GalnAsP LD on InP/Si substrate. Phys Status Solidi (A) 215, 1700357 (2018).

26. Tsushima K, Uchida K, Han X, Sugiyama H, Aikawa M et al. Lasing characteristics of GalnAsP SCH MQW high-mesa laser on silicon substrate. In 2019 24th OptoElectronics and Communications Conference (OECC) and 2019 International Conference on Photonics in Switching and Computing (IEEE, 2019); http://doi.org/10.23919/PS.2019.8818102.

27. Sugiyama H, Uchida K, Han X, Periyanayagam GK, Aikawa M et al. MOVPE grown GalnAsP/GalnAsP SCH-MQW laser diode on directly-bonded InP/Si substrate. J Cryst Growth 507, 93-97 (2019).

28. Hu YT, Liang D, Zhang C, Kurczveil G, Huang $X$ et al. Electrically-pumped $1.31 \mu \mathrm{m} \mathrm{MQW} \mathrm{lasers} \mathrm{by} \mathrm{direct} \mathrm{epitaxy} \mathrm{on} \mathrm{wafer-}$ bonded InP-on-SOI substrate. In 2018 IEEE Photonics Conference (IEEE, 2018); http://doi.org/10.1109/IPCon.2018.8527345

29. Hu YT, Liang D, Mukherjee K, Li YL, Zhang C et al. III/V-on-Si MQW lasers by using a novel photonic integration method of regrowth on a bonding template. Light Sci App/ 8, 93 (2019).

30. Hu YT, Liang D, Kurczveil G, Beausoleil RG. A bonded template-assisted monolithic integration platform. Proc SPIE 11184, 1118400 (2019).

31. Besancon C, Vaissiere N, Dupré C, Fournel F, Sanchez L et al. Epitaxial growth of high-quality AIGalnAs-based active structures on a directly bonded $\mathrm{InP}-\mathrm{SiO}_{2} / \mathrm{Si}$ substrate. Phys Status Solidi (A) 217, 1900523 (2020).

32. Besancon C, Cerulo G, Néel D, Vaissiere N, Make D et al. Comparison of AIGalnAs-based laser behavior grown on hybrid InP-SiO $2 / \mathrm{Si}$ and InP substrates. IEEE Photon Technol Lett 32, 469-472 (2020).

33. Liang D, Huang X, Kurczveil G, Fiorentino M, Beausoleil RG. Integrated finely tunable microring laser on silicon. Nat Photon 10, 719-722 (2016).

34. Norman JC, Jung D, Zhang ZY, Wan YT, Liu ST et al. A review of high-performance quantum dot lasers on silicon. IEEE
J Quantum Electron 55, 2000511 (2019).

35. Besancon C, Fanneau P, Néel D, Cerulo G, Vaissiere $\mathrm{N}$ et al. Laser array covering $155 \mathrm{~nm}$ wide spectral band achieved by selective area growth on silicon wafer. In 2020 European Conference on Optical Communications (IEEE, 2020); http://doi.org/10.1109/ECOC48923.2020.9333230.

36. Matsumoto K, Kishikawa J, Nishiyama T, Kanke T, Onuki Y et al. Room-temperature operation of GalnAsP lasers epitaxially grown on wafer-bonded InP/Si substrate. App/ Phys Express $\mathbf{9}$, 062701 (2016).

37. Kallarasan PG, Nishiyama T, Kamada N, Onuki Y, Shimomura $\mathrm{K}$. $1.5 \mu \mathrm{m}$ laser diode on InP/Si substrate by epitaxial growth using direct bonding method. In 2017 Conference on Lasers and Electro-Optics (IEEE, 2017); http://doi.org/10.1364/CLEO_AT.2017.JTu5A.108.

38. Aihara T, Hiraki T, Takeda K, Hasebe K, Fujii T et al. Lateral current injection membrane buried heterostructure lasers integrated on 200-nm-thick Si waveguide. In 2018 Optical Fiber Communications Conference and Exposition (IEEE, 2018); http://doi.org/10.1364/ofc.2018.w3f.4.

39. Moscoso-Mártir A, Merget F, Mueller J, Hauck J, RomeroGarcía S et al. Hybrid silicon photonics flip-chip laser integration with vertical self-alignment. In 2017 Conference on Lasers and Electro-Optics Pacific Rim (IEEE, 2017); http://doi.org/10.1109/CLEOPR.2017.8118971.

40. Zhang $\mathrm{C}$, Bowers JE. Silicon photonic terabit/s network-onchip for datacenter interconnection. Opt Fiber Technol 44, 2-12 (2018).

41. Huang R, Lan T, Li C, Li J, Wang ZY. Investigation of the blistering and exfoliation mechanism of GaAs wafers and $\mathrm{SiO}_{2} / \mathrm{Si}_{3} \mathrm{~N}_{4} / \mathrm{GaAs}$ Wafers by $\mathrm{He}^{+}$and $\mathrm{H}^{+}$implantation. Crystals 10, 520 (2020).

\section{Competing interests}

The authors declare no competing financial interests. 